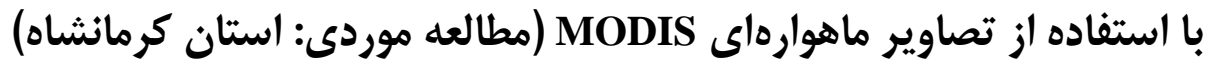

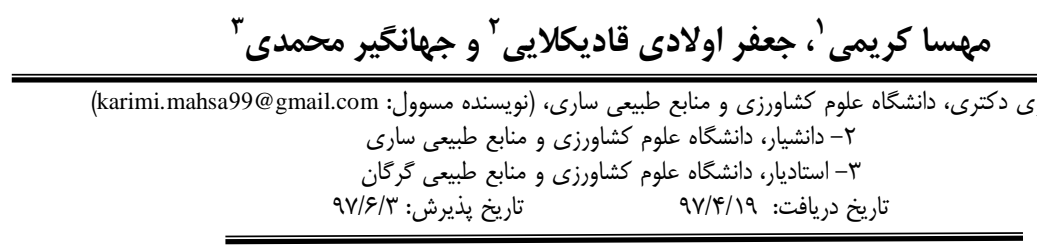

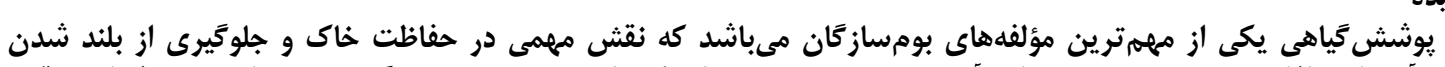

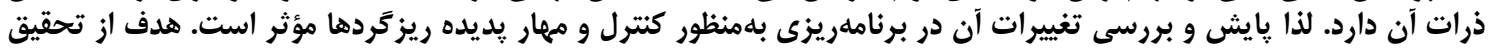

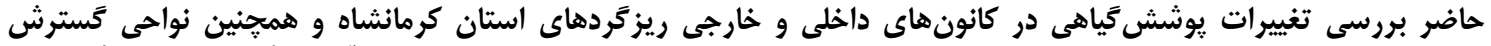

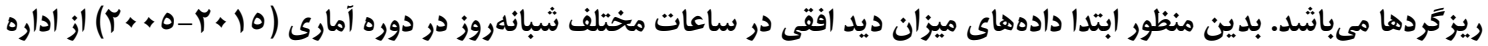

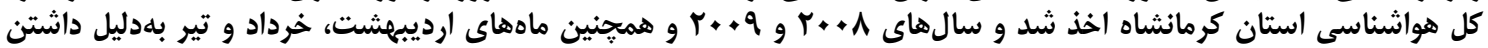

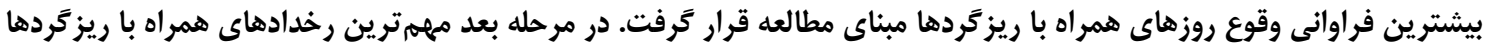

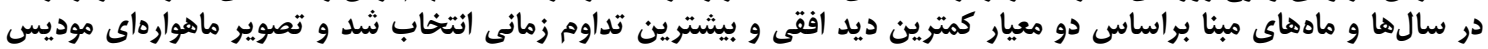

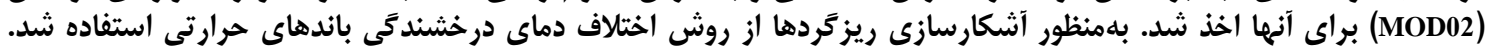

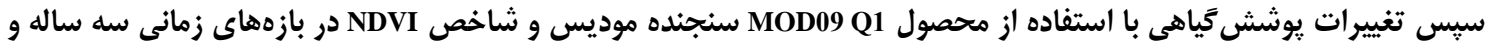

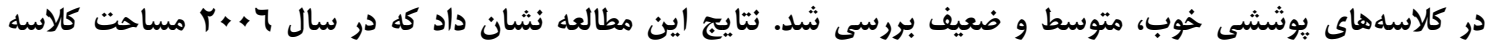

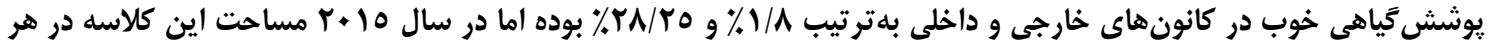

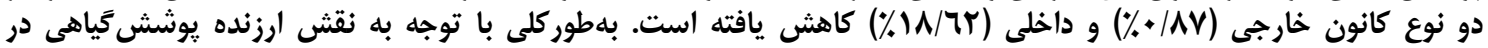

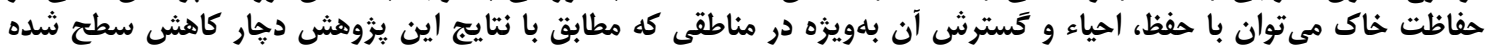

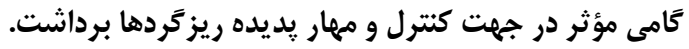

وازههاى كليدى: بوشش تياهى، NDVI MOD09 Q1، كانون ريزتر دها، اختلاف دماى درخشندىى

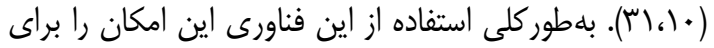

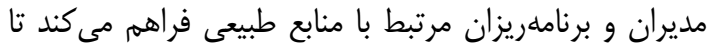

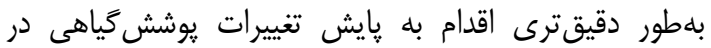

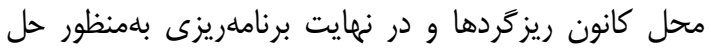

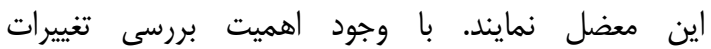

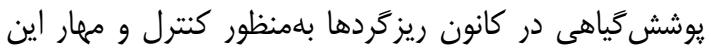

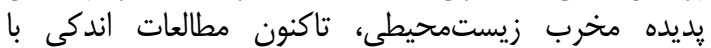

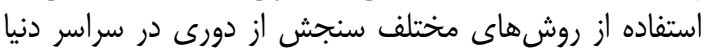

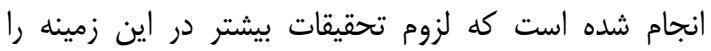

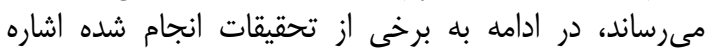

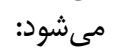

فرجزاده و كاوسى (^) روند تغييرات يوشش عياهى را در

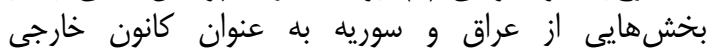

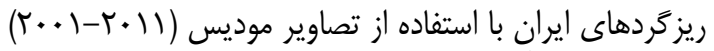

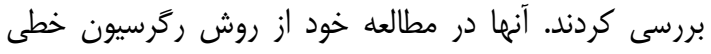

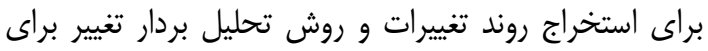

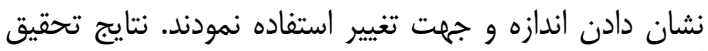

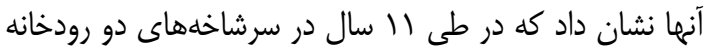

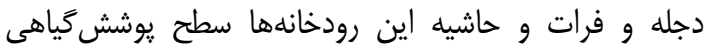

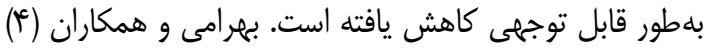

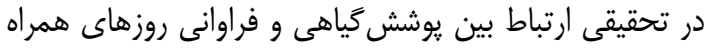

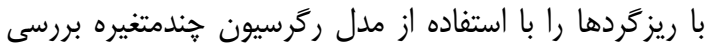

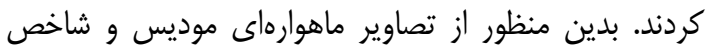

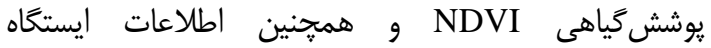

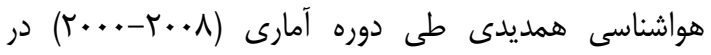

مقدمه - مقام

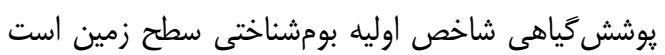

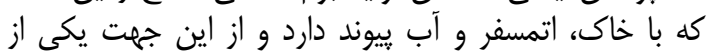

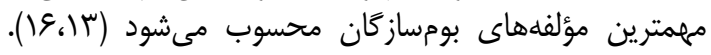

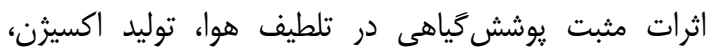

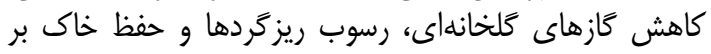

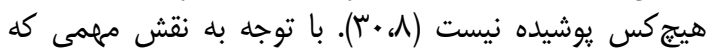

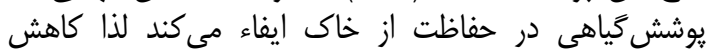

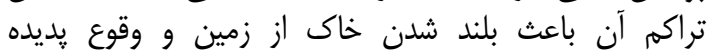

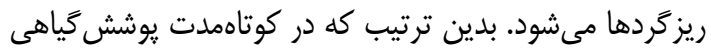

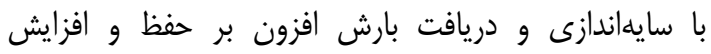

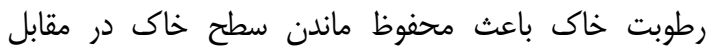

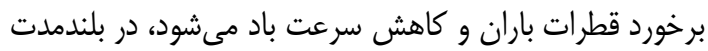

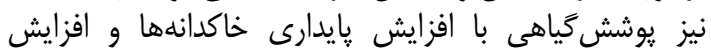

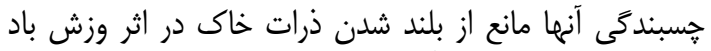

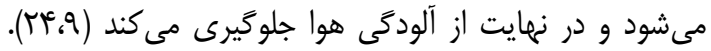

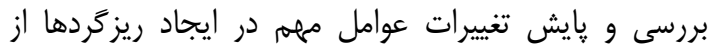

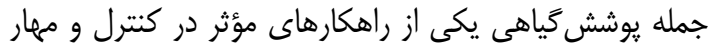

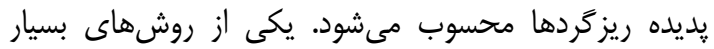

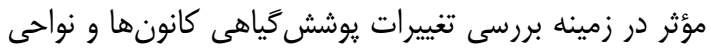

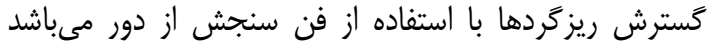

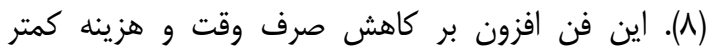

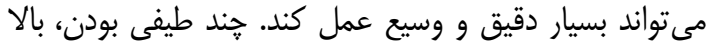

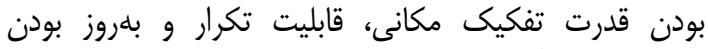

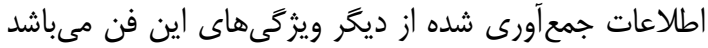




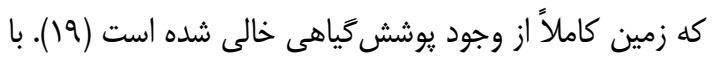

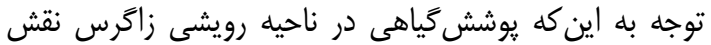

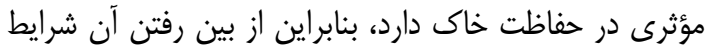

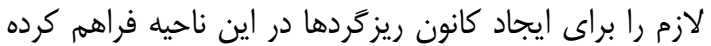

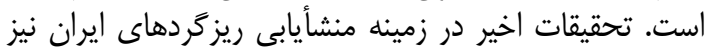

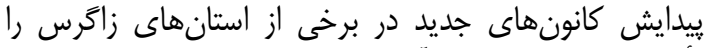

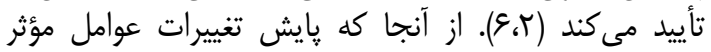

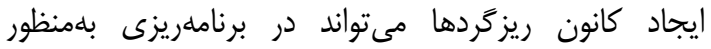

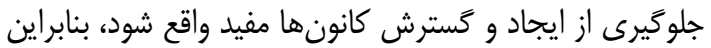

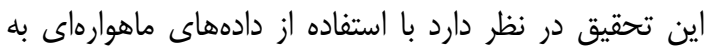

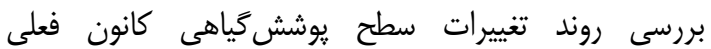

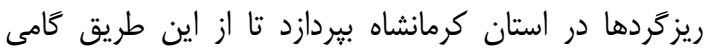
مؤثر در جهت حل اين معضل بردارد.

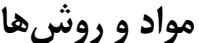
منطقه مورد مطالعه

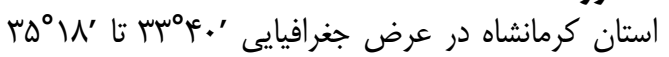

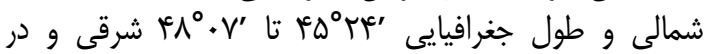

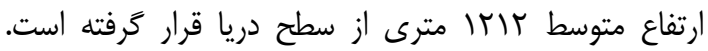

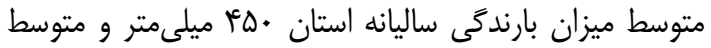

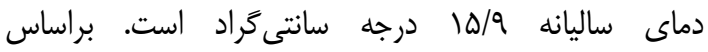
تقسيمبندى اقليمى دومارتن اقليم استان كرمانشاه دران دران خهار

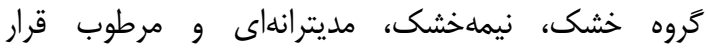

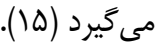
روش تحقيق در اين تحقيق ابتدا كانون ريزكردهاى استان كرمانشاه

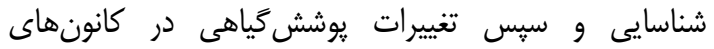

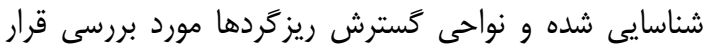

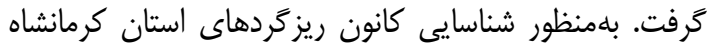

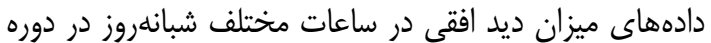

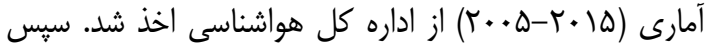

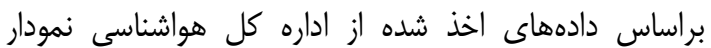

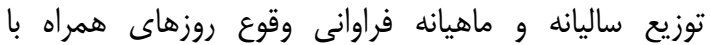

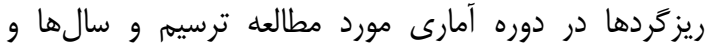

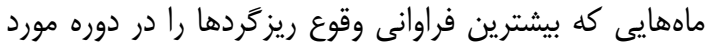

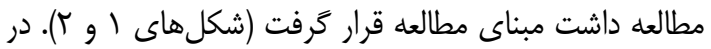

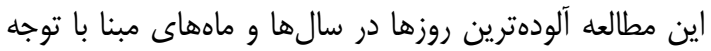

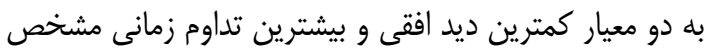

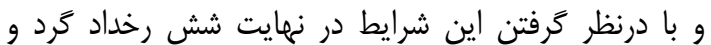

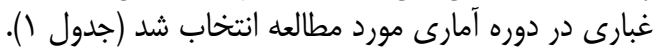

استان خوزستان استفاده شد. نتايج آنها نشان داد كه شاخص

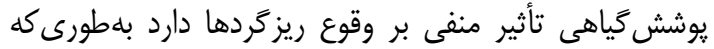

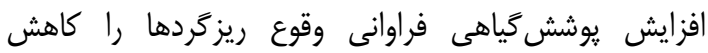

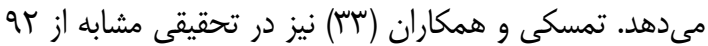

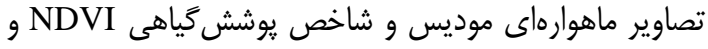

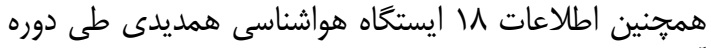

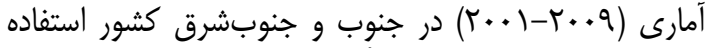

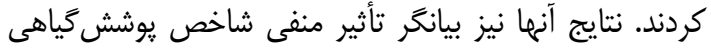

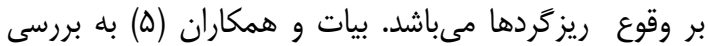

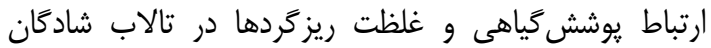

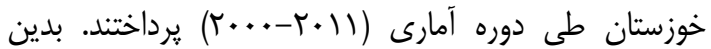

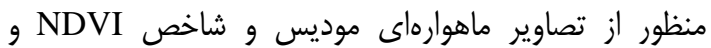

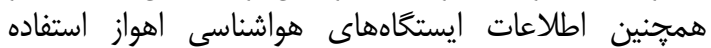

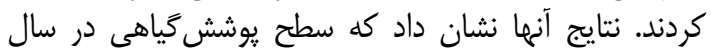

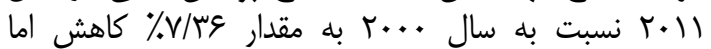

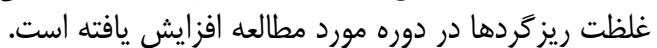

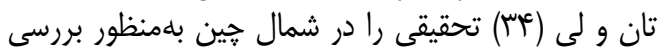

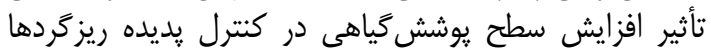

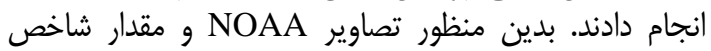

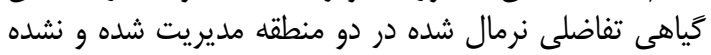

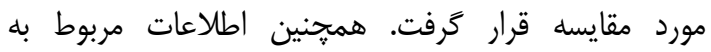

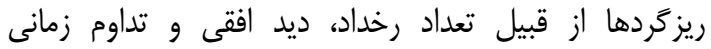

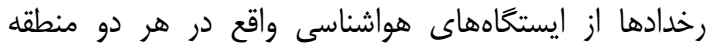

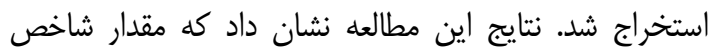

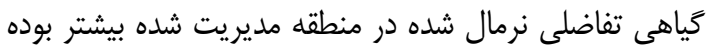

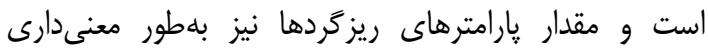
كاهش نشان داد. تحقيقات ديخر در همين زمين زمينه بار با استفاده از

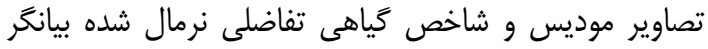

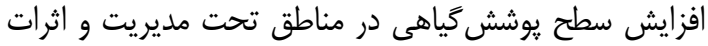

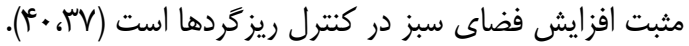

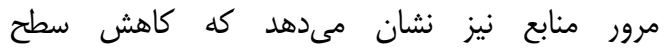
يوشش گياهى نقش مؤثرى در وقوع ريزَّردها ايفاء مى كند، از

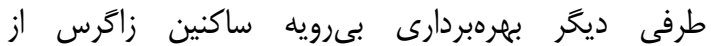

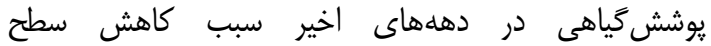
يوشش

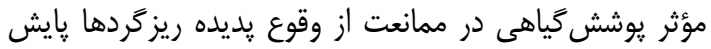

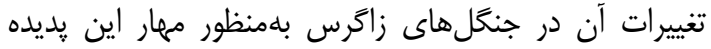

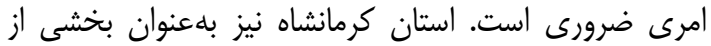

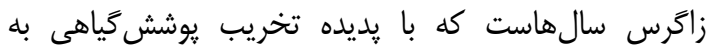

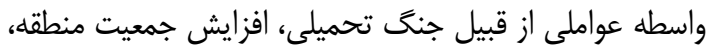

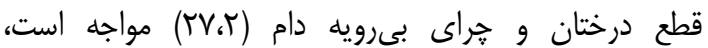

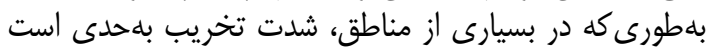


جدول ا- مهمترين رخدادهاى همراه با ريزگردها براساس ديد افقى و فراوانى وقوع در سال ها و ماههاى مبناى مطالعه Table 1. The most important of the dusty days based on horizontal visibility and frequency of occurrences

\begin{tabular}{|c|c|c|c|}
\hline فراوانى وقوع & ديد افقى (متر) & تاريخ & رديف \\
\hline$r$ & $\Lambda \cdots$ & $r \cdot \cdot N / \cdot \Delta / \lambda \Lambda$ & 1 \\
\hline f & $1 .$. & $r \cdot \cdot N / \cdot g / 19$ & $r$ \\
\hline 9 & $r .$. & $r \cdot \cdot N / \cdot V / \cdot \Lambda$ & r \\
\hline r & $q .$. & $r \cdot . q / \cdot \Delta / \cdot r$ & c \\
\hline$\wedge$ & $\Lambda .$. & $r . . q / .9 / 19$ & $\Delta$ \\
\hline$\wedge$ & $r .$. & $r \cdot . q / \cdot v / l f$ & 8 \\
\hline
\end{tabular}

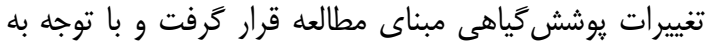

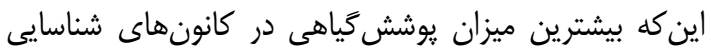

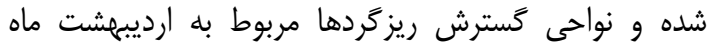

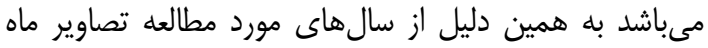

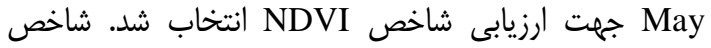

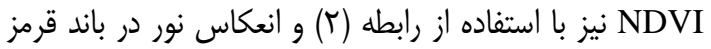
(باند () و مادون قرمز نزديك (باند r) محاسبه شد.

$(\mathrm{NIR}-\mathrm{RED}) /(\mathrm{NIR}+\mathrm{RED})$

رابطه (r)

كه در آن NIR: انعكاس نور در باند مادون قرمز نزديك، RED دNDVI

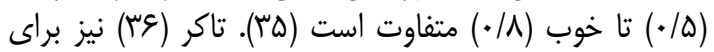

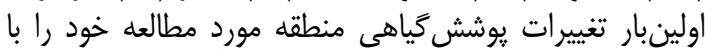

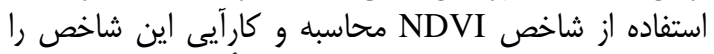

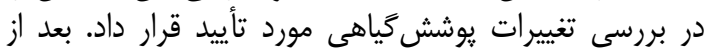

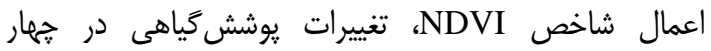

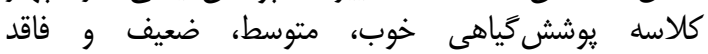

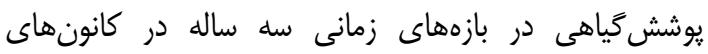

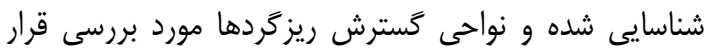

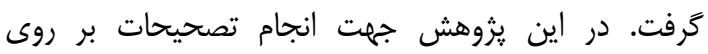

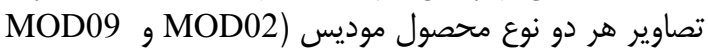

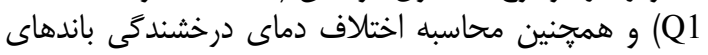

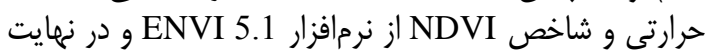
جهت طبقلهندى نقشهها و خروجى گر خرفتن از نرمافزار 10.3 ArcGIS در اين مطالعه بررسى نمودار توزيع ساليانه و ماهيانه

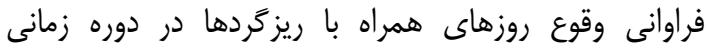

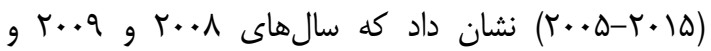

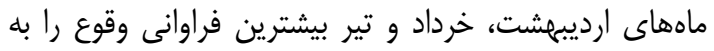

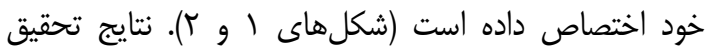

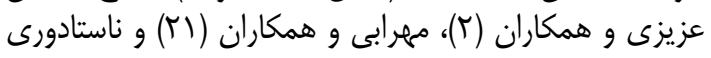

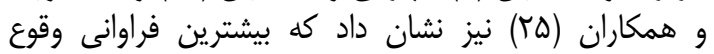

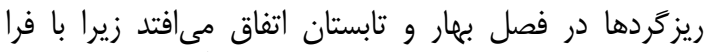

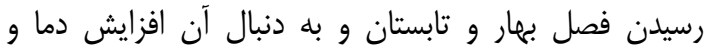

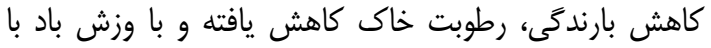

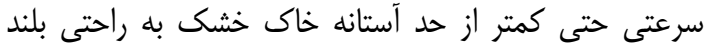

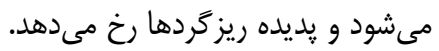

در اين تحقيق بلمنظور آشكارسازى ريزگردها از تصاوير سطح

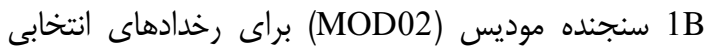

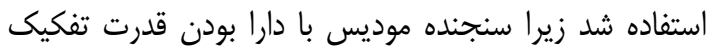

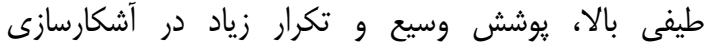

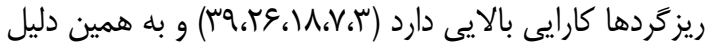

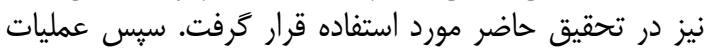

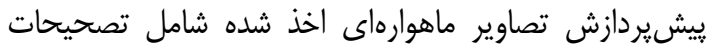

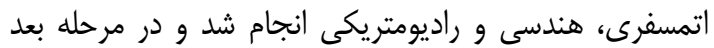

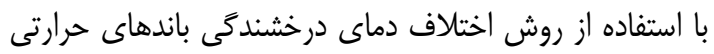

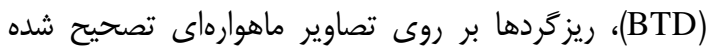

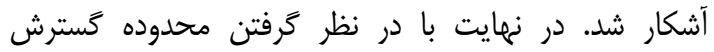

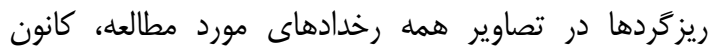

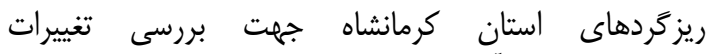

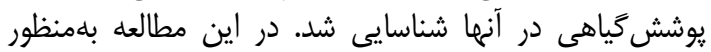

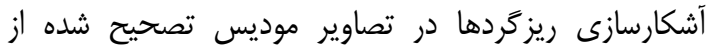

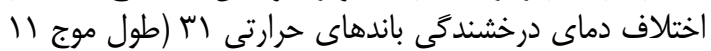

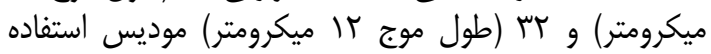

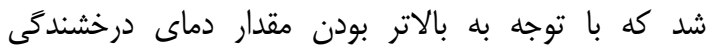

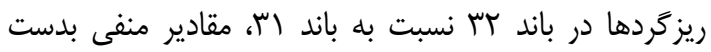

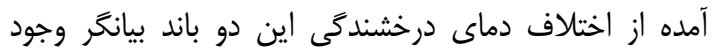

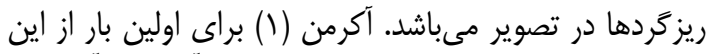

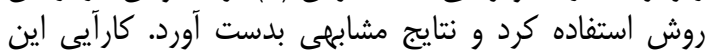

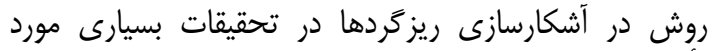

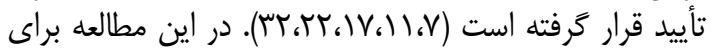

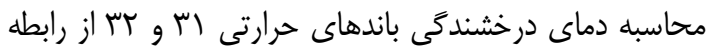

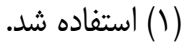

$$
B T_{i}=\frac{h c}{\lambda_{i} k \ln \left(\frac{2 h c^{2}}{l\left(\lambda_{i}\right) \lambda_{i}{ }^{5}}+1\right)}
$$

كه در آن BT

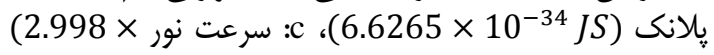

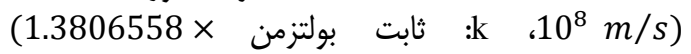

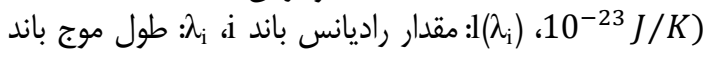

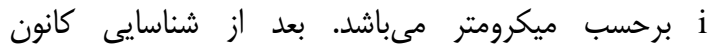

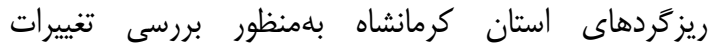
يوشش ريزگردها از محصول MOD09 Q1 سنجنداه درد موديس استفاده

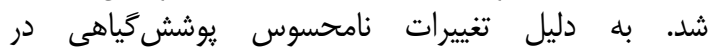

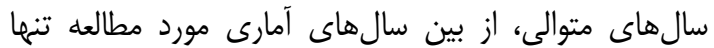

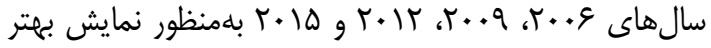




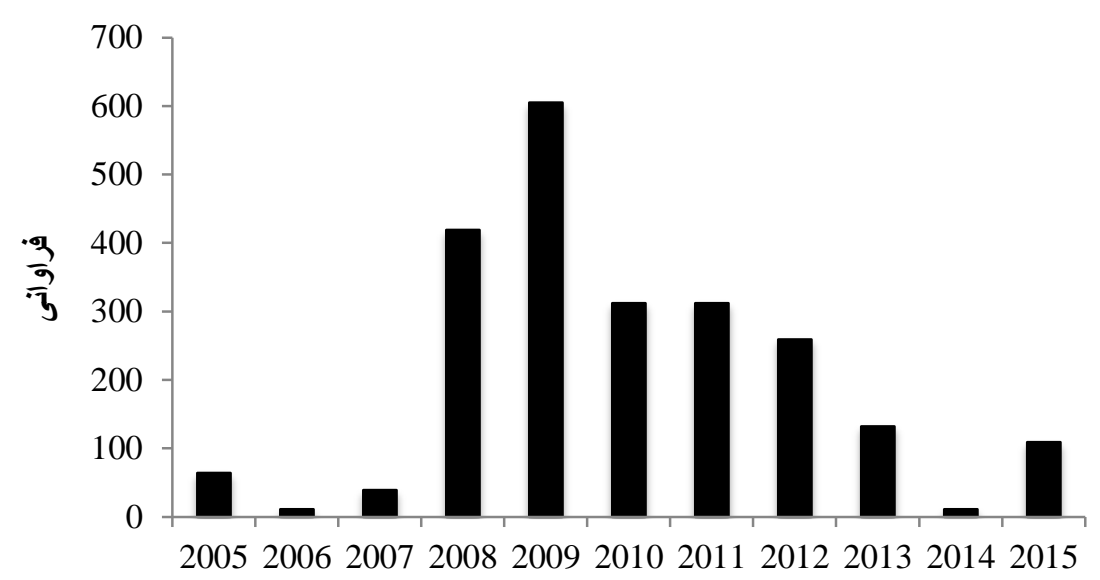

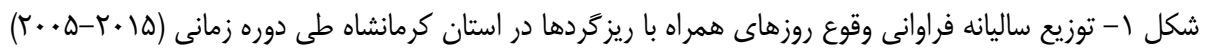

Figure 1. Yearly distribution of frequency of occurrence of dusty days in Kermanshah province during the 2005 to 2015

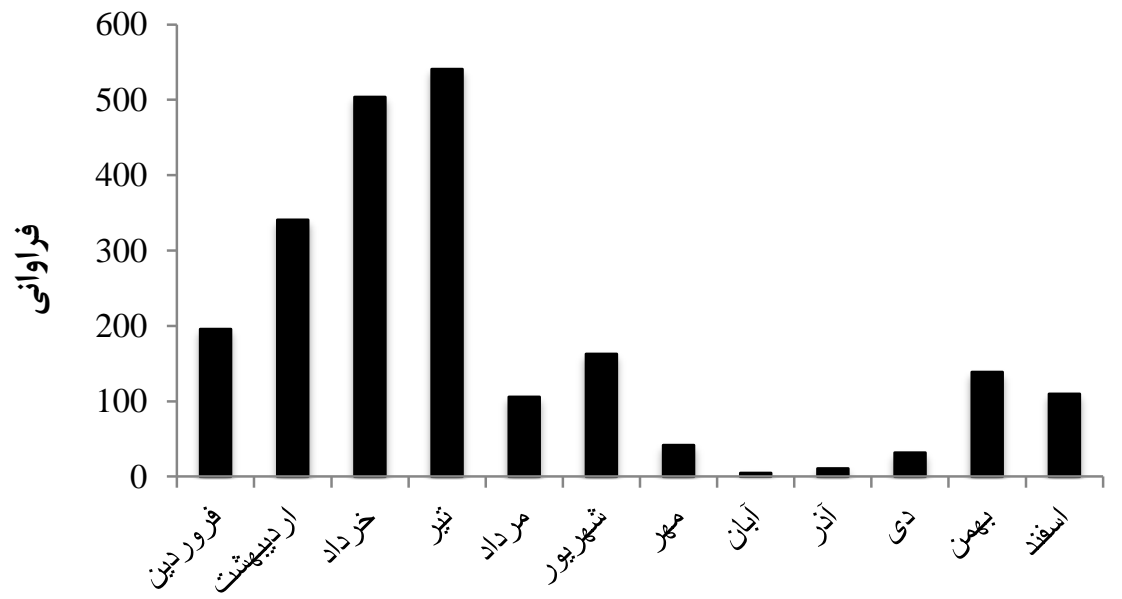

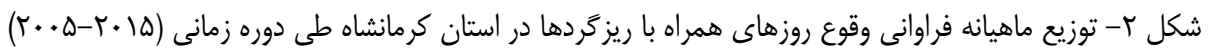

Figure 2. Monthly distribution of frequency of occurrence of dusty days in Kermanshah province during the 2005 to 2015

رخدادهاى گرد و غبارى سال 9+ +.r شرق سوريه، بخشهايى

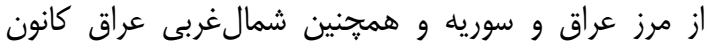

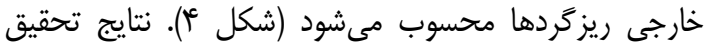

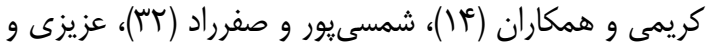

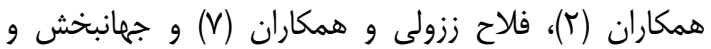

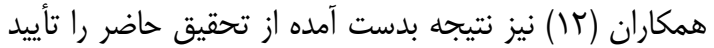

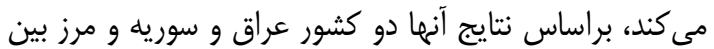

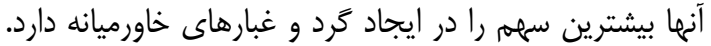

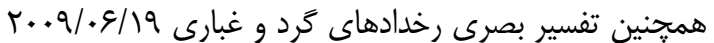

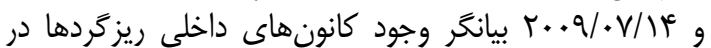

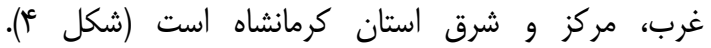

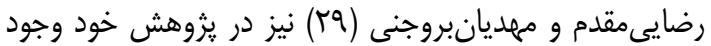

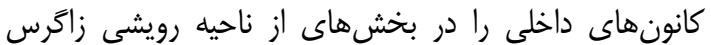
تأييد كرد.
در شكلهاى س و ع تصاوير روزهاى همراه با ريزگردها

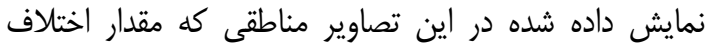

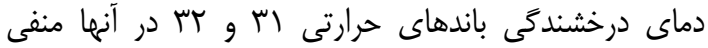

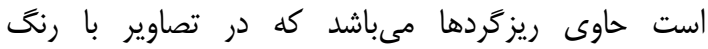

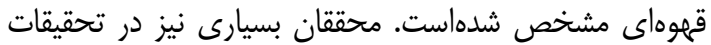

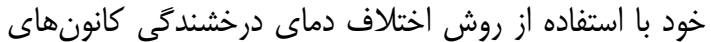

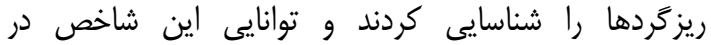

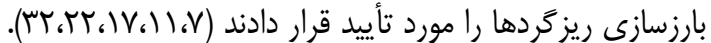

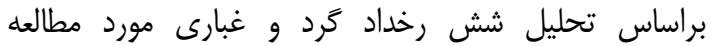

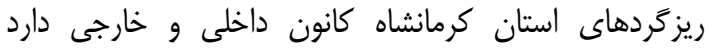

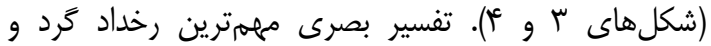

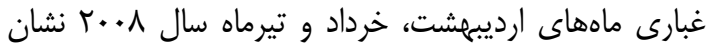

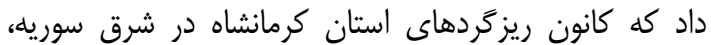

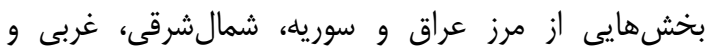

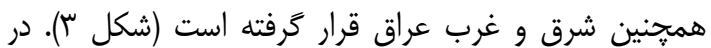



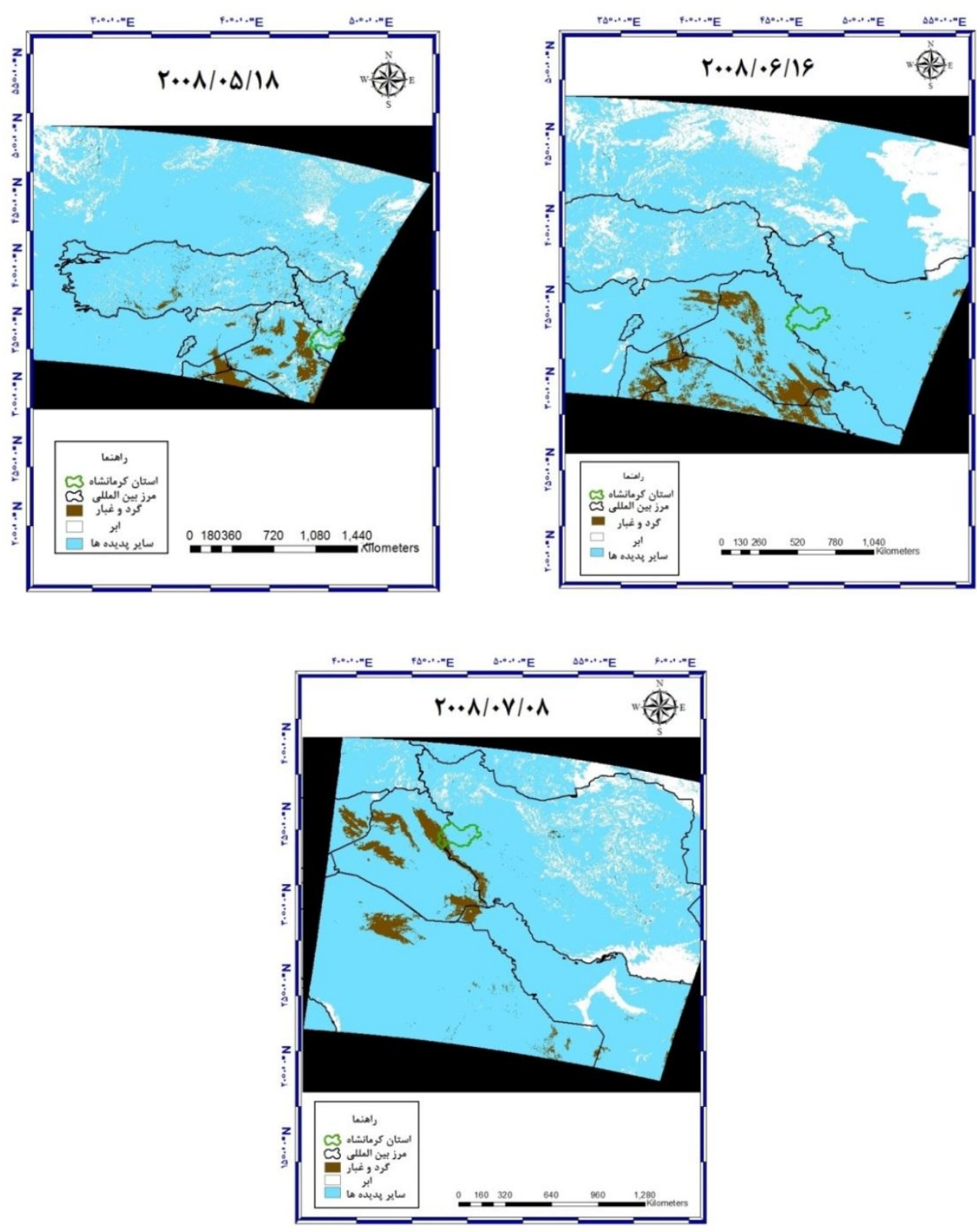

شكل س- آشكارسازى كانونها و محدوده كسترش ريزگَردها با استفاده از روش اختلاف دماى درخشندگى در تصاوير موديس روزهاى

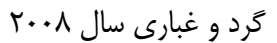

Figure 3. Detecting dust sources and extension area using BTD method with MODIS Image in 2008 

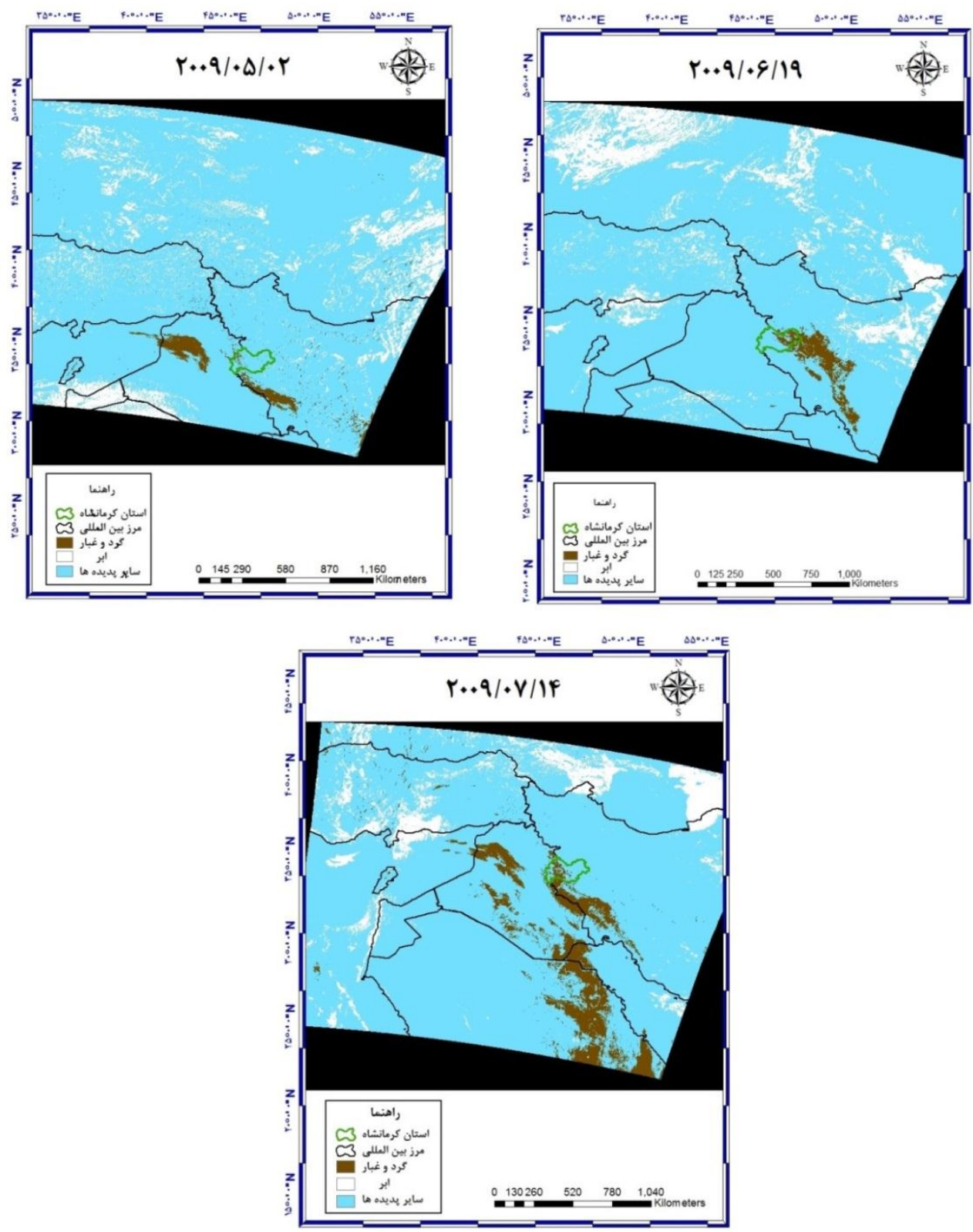

شكل ع- آشكارسازى كانونها و محدوده كَترش ريزكَردها با استفاده از روش اختلاف دماى درخشندَى در تصاوير موديس روزهاى كَرد و غبارى

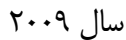

Figure 4. Detecting dust sources and extension area using BTD method with MODIS Image in 2009

يوشش يُاهى در آنها مشخص شده است. بررسى نقشه

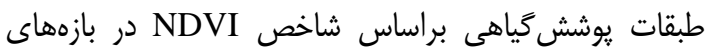

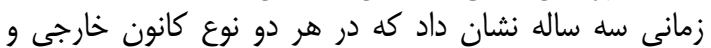

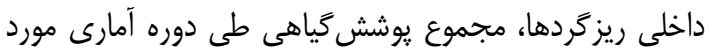

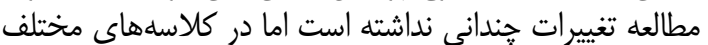

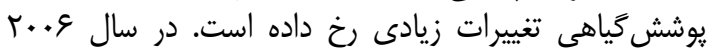

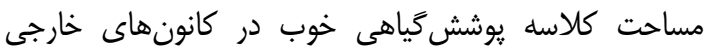

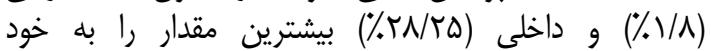

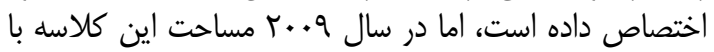

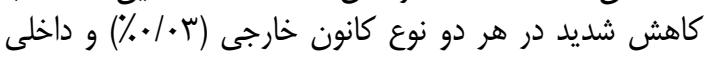

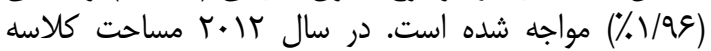

بعد از شناسايى كانونها و محدوده گسترش ريزگردها

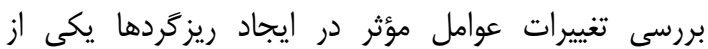

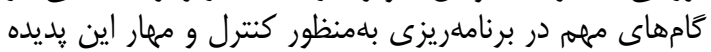

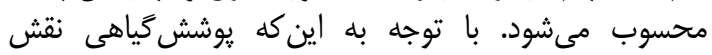

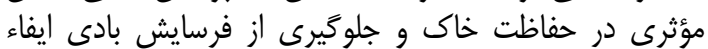

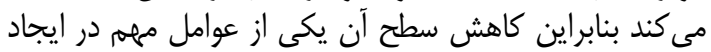

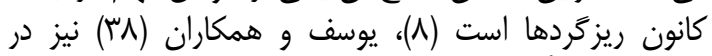

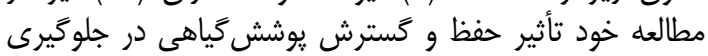

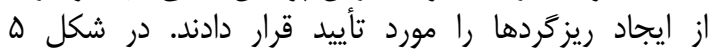

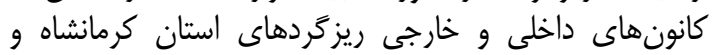

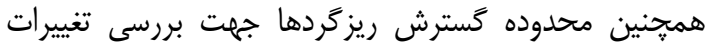


كانونهاى خارجى با كذشت زمان كاهش يافته است. اين

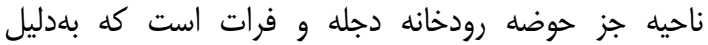

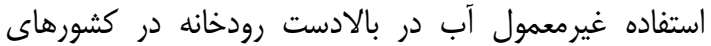

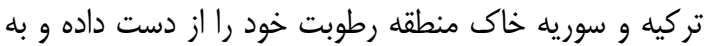

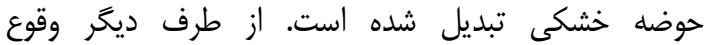

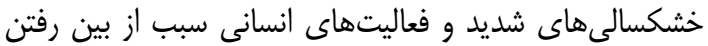

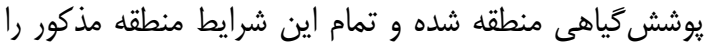

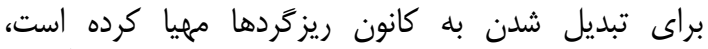

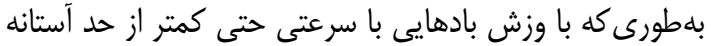

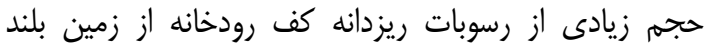

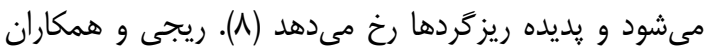

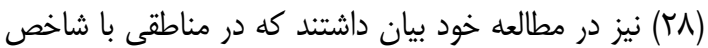

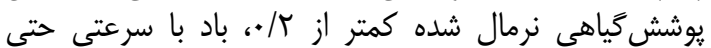

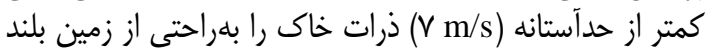

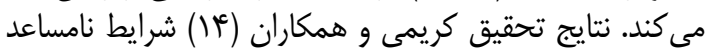

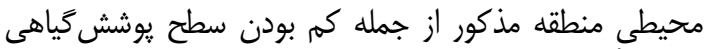

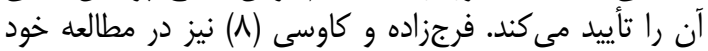

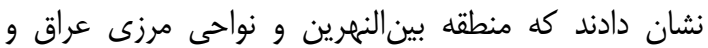

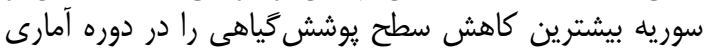

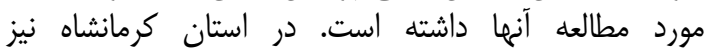

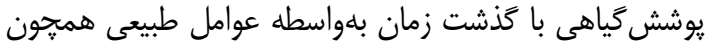

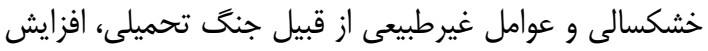

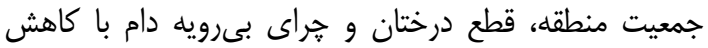

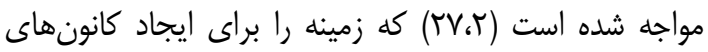

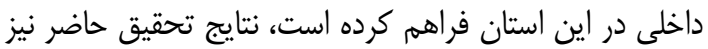

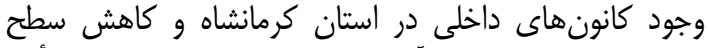

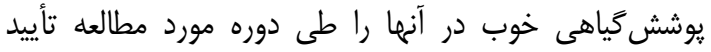

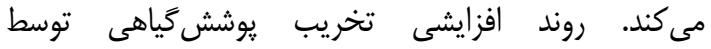

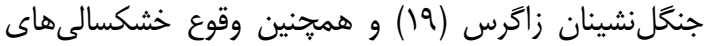

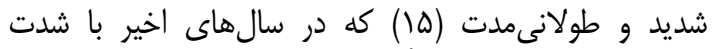

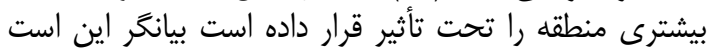

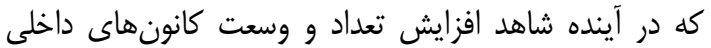

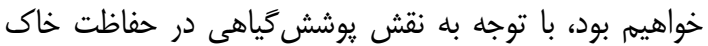

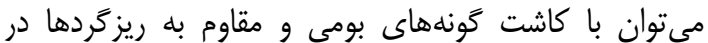

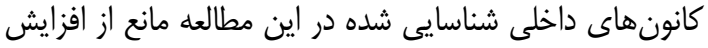

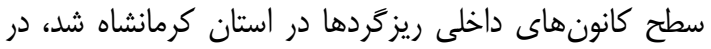

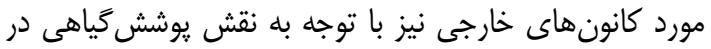

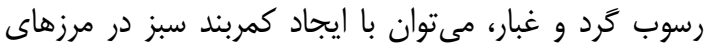

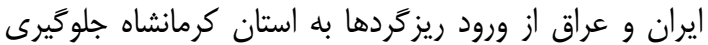

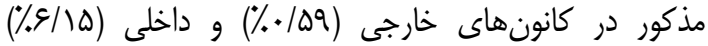

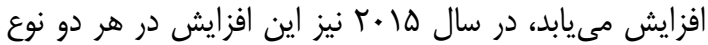

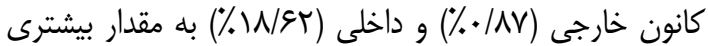

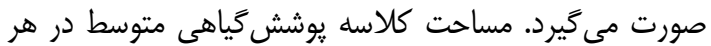

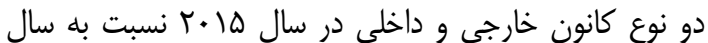

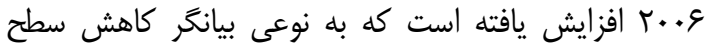

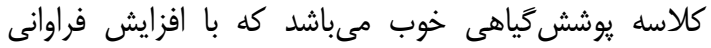

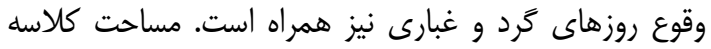

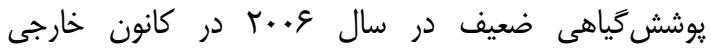

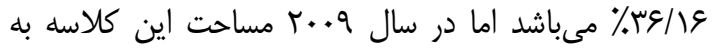

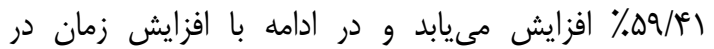

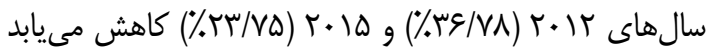

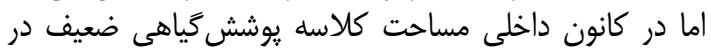

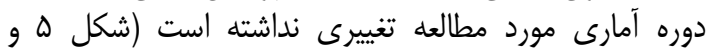

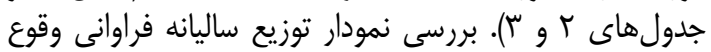

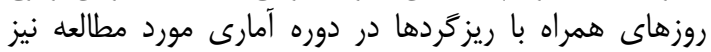

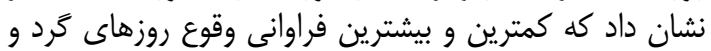

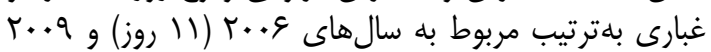

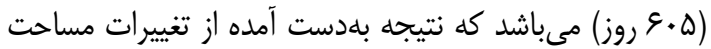

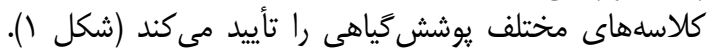

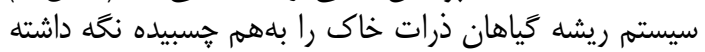

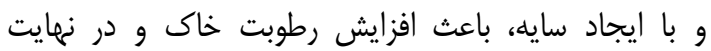

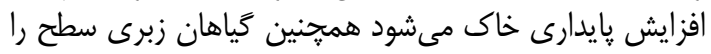

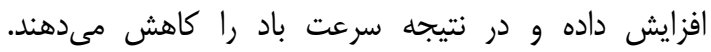

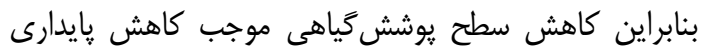

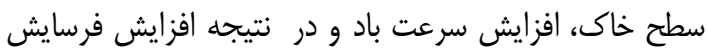

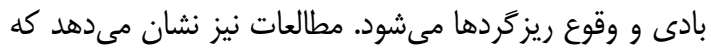

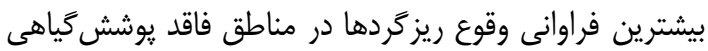

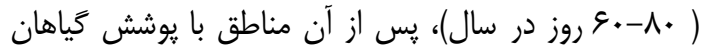

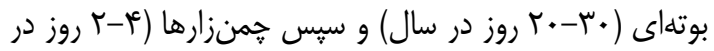

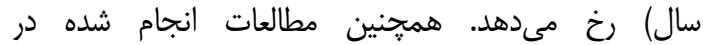

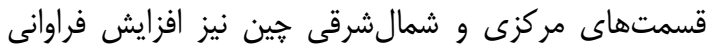

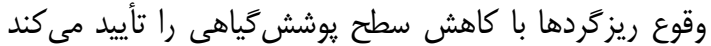

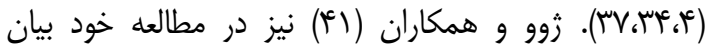

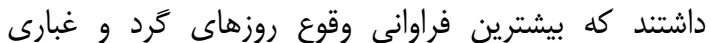

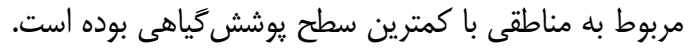

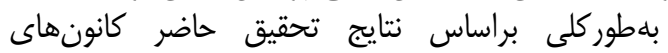

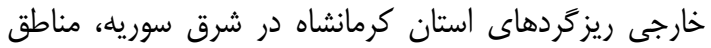

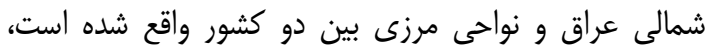

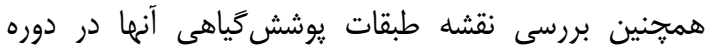

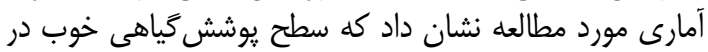



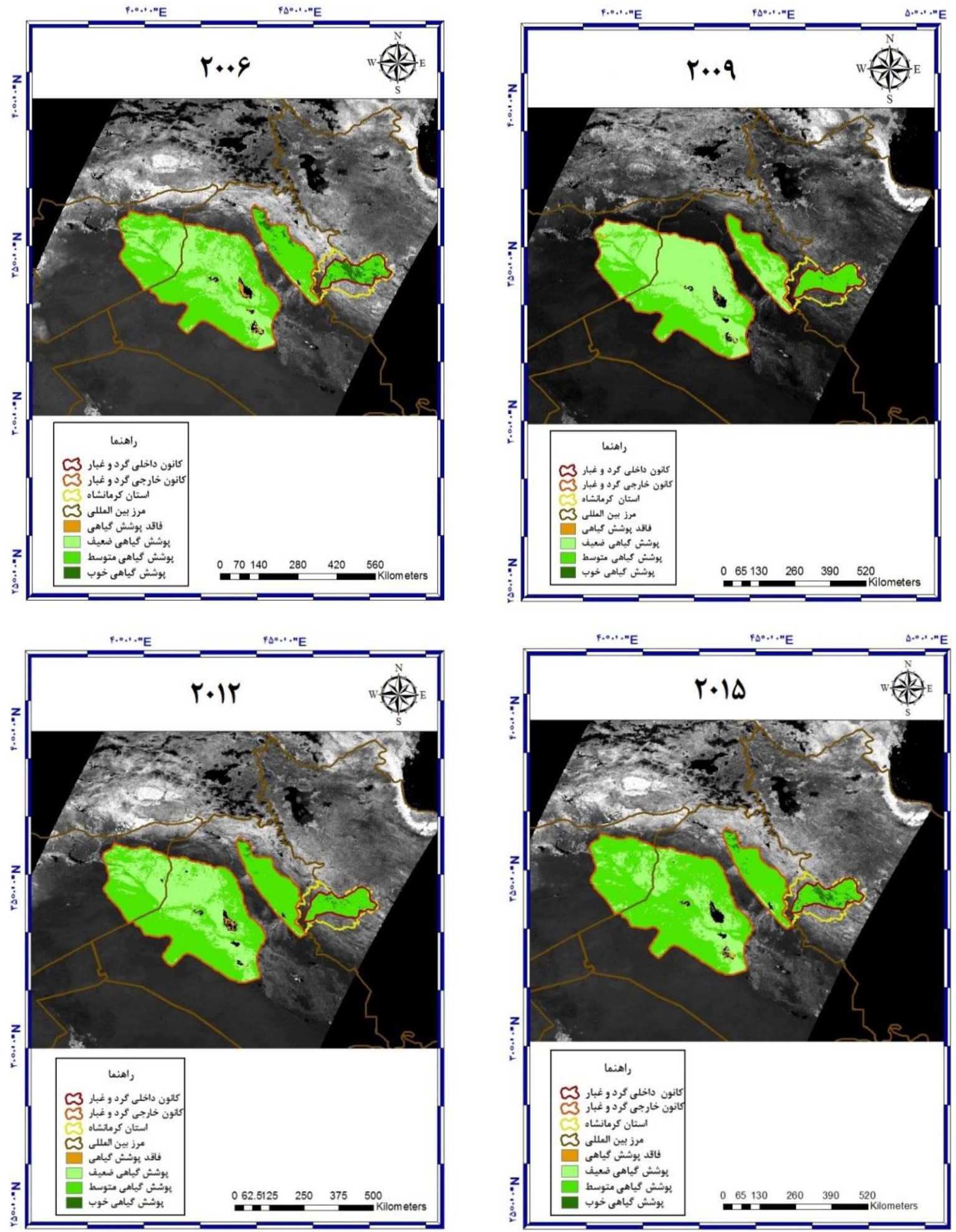

شكل ه- نقشه طبقات يوشش گياهى در كانونهاى داخلى و خارجى ريزَّردهاى استان كرمانشاه و نواحى كَسترش ريزكَردها

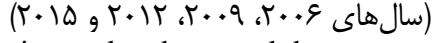

Figure 5. Map of vegetation classes in the internal and external dust storm sources of Kermanshah province and dust extension areas $(2006,2009,2012$, and 2015) 
جدول r- مساحت طبقات يوشش Table 2. The area of vegetation classes in the external dust storm sources of Kermanshah province

\begin{tabular}{|c|c|c|c|c|c|}
\hline فاقد يوشش כياهى (\%) & مجموع سطح :وشش كياهى (\%) & يوشش كياهى ضعيف (\%) & يوشش كياهى متوسط (\%) & يوشش كياهى خوب (\%) & \\
\hline $1 / \mathrm{IV}$ & q//Ar & r & $9 \cdot / \mathrm{AV}$ & $1 / \Lambda$ & $r . . q$ \\
\hline$\cdot / N 1$ & $৭ 9 / / 9$ & $\Delta q / 4)$ & $r q / \wedge \Delta$ &.$/ . r$ & r...q \\
\hline.$/ V 9$ & $99 / T^{c}$ & rg/vı & $91 / A V$ & .109 & $r \cdot I r$ \\
\hline$\cdot / V \Delta$ & ११//ब & $r \mu / v \Delta$ & $V E / q \mu$ & $\cdot /$ AV & $r \cdot 10$ \\
\hline
\end{tabular}

جدول س- مساحت طبقات يوشش گياهى در كانون داخلى ريزگردهاى استان كرمانشاه Table 3. The area of vegetation classes in the internal dust storm sources of Kermanshah province

\begin{tabular}{|c|c|c|c|c|c|}
\hline فاقد يوشش كياهى (\%) & مجموع سطح يوشش گياهى (\%) & يوشش كياهى ضعيف (\%) & يوشش كياهى متوسط (\%) & يوشش گياهى خوب (\%) & \\
\hline.$/ \cdot \mu$ & $99 / 9 V$ & $\cdot / T$ & $V / \Delta T$ & $T N / T Q$ & r..G \\
\hline r.|. & $99 / 9 \vee$ & $\cdot / r$ & qV/A) & $1 / 98$ & $r . . q$ \\
\hline . & ११/१४ & $\cdot / 4$ & $q \pi / g r$ & $S / 10$ & $r .1 T$ \\
\hline .1 .9 & १९/१४ &.$/ r$ & $\Lambda / / T$ & IN/GT & $r \cdot 10$ \\
\hline
\end{tabular}
Geophysical Research, 102: 17069-17080.

2. Azizi. Gh.. A.A. Shamsibour. M. Miri and T. Safarrad. 2012. Statistical analysis-synoptic phenomenon of dust in the western half of Iran. Ecology, 38(3): 123-134 (In Persian).

3. Baddock, M.C., J.E. Bullard and R.G. Bryant. 2009. Dust source identification using MODIS: A comparison of techniques applied to the Lake Eyre Basin Australia. Remote Sensing of Environment, 113: 1511-1528.

4. Bahrami, H.A., M. Jalali, A. Darvishi Bolorany and R. Azizi. 2013. The spatial modeling of the time of storm storms in Khuzestan province. Iranian Remote Sensing and GIS, 5(2): 95-114 (In Persian).

5. Bayat, R., S. Jafari, B. Ghermezcheshmeh and A.H. Charkhabi. 2016. Studying the effect of dust on vegetation changes (case study: Shadegan Wetland, Khuzestan). RS and GIS for Natural Resources, 7(2): 17-32 (In Persian).

6. Cao, H., J. Liu, G. Wang, G. Yang and L. Luo. 2015. Identification of sand and dust storm source areas in Iran. Journal of Arid Land, 7(5): 567-578.

7. Fallah Zzooli, M., A. Vafaei Nezhad, M.M. Kheirkhah Zarkesh and F. Ahmadi Dehkae. 2014. Synoptic monitoring and analysis of dust phenomena using remote sensing and GIS (case studv: Dust 18 June 2012). Geographical Information Scientific-Research Quarterly Periodical, 23(91): 69-80 (In Persian).

8. Frarajzadeh, M. and M. Kavosi. 2014. The evaluation of vegetation variations trend using linear regression methods and change vector analysis. Geography and Environmental Planning, 25(4): 6982 (In Persian).

9. Godı'nez-Alvarez, H., J.E. Herrick, M. Mattocks, D. Toledo and J. Van Zee. 2009. Comparison of three vegetation monitoring methods: their relative utility for ecological assessment and monitoring. Ecological Indicators, 9: 1001-1008.

10. Hua, N.P., F. Kobayashi, Y. Iwasaka, G.Y. Shi and T. Naganuma. 2007. Detailed identification of desertoriginated bacteria carried by Asian dust storms to Japan. Aerobiologia, 23(4): 291-298.

11. Huang, J., J. Ge and F. Weng. 2007. Detection of Asian dust storms using multisensor satellite easurements. Remote Sensing Environment, 110: 186-191.

12. Jahanbakhsh. S.. Kh. Valizadeh. M. Khosravi. B. Zeinali and S. Asghari. 2014. Identifving and detecting the Hurricane of Iran 1 July 2008 using the MODIS sensor. Geographical Space ScientificResearch Quarterly Periodical, 46(14): 31-50 (In Persian).

13. Jensen, R., J. Gatrell, J. Boulton and B. Harper. 2004. Using remote sensing and geographic information systems to study urban quality of life and urban forest amenities. Ecology and Society, 9(5): 5.

14. Karimi. Kh.. H. Taheri Shahr Aeini. M. Habibi Nokhandan and N. Hafezi Moghadas. 2011. Identifying the origins of dust storm production in the Middle East using remote sensing. Journal of Clinical Research, 2(7-8): 57-72 (In Persian).

15. Karimi, M., K. Shahedi and M. Byzedi. 2015. Analysis of hydrological drought using constant threshold level method (case study: Karkheh River Basin, Iran). Journal of Watershed Management Research, 6(11): 59-72 (In Persian).

16. Krishna, T.M., G. Ravikumar and M. Krishnaveni. 2009. "Remote sensing based agricultural drought assessment in Palar Basin of Tamil Nadu State, India". Journal of the Indian Society of Remote Sensing, 37: 9-20.

17. Li, X., L. Ge, Y. Dong and H.C. Chang. 2010. Estimating the greatest dust storm in eastern Australia with MODIS satellite images. Published by IEEE.

18. Madhavan, S., J.J. Qu and X. Hao. 2017. Saharan dust detection using multi-sensor satellite measurements. Heliyon, http://dx.doi.org/10.1016/j.heliyon.2017.e00241.

19. Marvie Mohadjer, M.R. 2007. Silviculture. Second Edition, University of Tehran Press, 387 pp (In Persian). 
20. Masrouri, E., Sh. Shataei, M.H Moayeri, J. Soosani and R. Bagheri. 2015. Modeling of forest degradation extend using physiographic and socio-economic variables (case study: A part of KakaReza District in Khoram-Abad). Ecology of Iranian Forests, 3(5): 20-30 (In Persian).

21. Mehrabi, Sh., S. Soltani and R. Jafari. 2015. Investigating the relationship between climatic varameters and occurrence of dust. Journal of Agricultural Science and Technology, Water and Soil Science, 19(71): 69-80 (In Persian.)

22. Mie, D., L. Xiushan, S. Lin and W. Ping. 2008. A dust-storm process dynamic monitoring with multitemporal MODIS data. The International Archives of Photogrammetry, Remote Sensing and Spatial Information Sciences, 10(7): 965-969.

23. Mirzaei Zadeh, V., A. Mahdavi, A. Karmshahi and A.A. Jaefarzadeh. 2015. Application of an integrated CA-Markov model in simulating spatiotemporal changes in forest cover: a case study of Malekshahi county forests, Ilam province. Ecology of Iranian Forests, 3(5): 42-52 (In Persian).

24. Munson, S.M., R.H. Webb and A. Hubbard. 2011. A comparison of methods to assess longterm changes in Sonoran desert vegetation. Journal of Arid Environments, 75: 1228-1231.

25. Nastagdori, L., D. Jugder and Y. Schung. 2002. Analysis of dust storms observed. Mongolia during 1937-1999, $12 \mathrm{pp}$

26. Ochirkhuyag, L. and R. Tsolmon. 2008. Monitoring the source of trans-national dust storms north east Asia. The international archives of the photogrammetry, Remote Sensing and Spatial Information Sciences, 10(7): 835-840.

27. Pourreza, M., J.D. Shaw and H. Zangeneh. 2008. Sustainability of Wild Pistachio (Pistacia atlantica Desf.) in Zagros forests, Iran. Forest Ecology and Management, 225: 3667-3671.

28. Reiji, K., L. Bai and J. Wang. 2009. Relationships among dust outbreaks, vegetation cover, and surface soil water content on the Loess Plateau of China, 1999-2000. Catena. 77(3): 292-296.

29. Rezaei Moghadam. M.H. and M. Mahdian Borouieni. 2015. Determination of the dust using NOAA satellite AVHRR (case study: South-West of Iran). Geography and Environmental Sustainability, 5(17): 1-13 (In Persian).

30. Sauer, T. and J.D. Ries. 2008. Vegetation cover and geomorphodynamics on abandoned fields in the Central Ebro Basin (Spain). Geomorphology, 102: 267-277.

31. Scheer, L. and R. Sitko. 2007. Assessment of some forest characteristics employing IKONOS satellite data. Journal of Forest Science, $53 \mathrm{pp}$.

32. Shamsipour, A.A. and T. Safarrad. 2012. Satellite and synoptic analysis of dust storm in western half of Iran (case studv: Julv 2009). Natural Geographv Research. 44(79): 111-126 (In Persian).

33. Tamassoki, E., A. Khoorani, A. Dervishi Bolorany and A. Nohegar. 2016. Monitoring and predicting the occurrence of dust storms using Dwarves data, spatial information system and terrestrial data based on the study of changes in vegetation and climatic elements (case study: south and south east of Iran). Iranian Remote Sensing and GIS, 7(4): 27-44 (In Persian).

34. Tan, M. and X. Li. 2015. Does the green great wall effectively decrease dust storm intensity in China? a study based on NOAA NDVI and weather station data. Land Use Policy, 43: 42-47.

35. Thenkabail, P.S., R.B. Smith and E. De Pauw. 2002. Evaluation of narrow band and broadband vegetation indices for determining optimal hyper spectral wavebands for agricultural crop characterization. Photogrammetric Engineering and Remote Sensing, 68(6): 607-621.

36. Tucker, C.J. 1979. Red and photographic infrared linear combinations for monitoring vegetation. Remote Sensing of Environment, 8(2): 127-150.

37. Wu, Zh., J. Wu, J. Liu, B. He, T. Lei and Q. Wang. 2013. Increasing terrestrial vegetation activity of ecological restoration program in the Beijing-Tianjin sand source region of China. Ecological Engineering, 52: 37-50.

38. Youssef, F., G. Erpul, P. Bogman, W.M. Cornelis and D. Gabriels. 2008. Determination of efficiency of Vaseline slide and Wilson and Cooke sediment traps by wind tunnel experiments. Environmental Geology, 55(4): 741-750.

39. Yue, H., Ch. He, Y. Zhao, Q. Ma and Q. Zhang. 2017. The brightness temperature adjusted dust index: An improved approach to detect dust storms using MODIS imagery. International Journal of Applied Earth Observation and Geoinformation, 57: 166-176.

40. Zhang, Y., Ch. Peng, W. Li, L. Tian, Q. Zhu, H. Chen, X. Fang, G. Zhang, G. Liu, X. Mu, Zh. Li, Sh. Li, Y. Yang, J. Wang and X. Xiao. 2016. Multiple afforestation programs accelerate the greenness in the 'Three North' region of China from 1982 to 2013. Ecological Indicators, 61(2): 404-412.

41. Zhou, X.K., P.M. Zhai. 2004. Relationship between vegetation coverage and spring dust storms over northern China. Geophysical Research, 109: 987-993. 


\title{
Investigating the Vegetation Changes in the Internal and External Dust Storm Sources using MODIS Satellite Imagery (Case Study: Kermanshah Province)
}

\author{
Mahsa Karimi $^{1}$, Jafar Oladi Ghadikolaei ${ }^{2}$ and Jahangir Mohammadi ${ }^{3}$ \\ 1- Ph.D. Student, Sari Agricultural Sciences and Natural Resources University, \\ (Corresponding author: karimi.mahsa99@gmail.com) \\ 2- Associate Professor, Sari Agricultural Sciences and Natural Resources University \\ 3- Assistant Professor, Gorgan Agricultural Sciences and Natural Resources University \\ Received: July 10, 2018 \\ Accepted: August 25, 2018
}

\begin{abstract}
Vegetation is one of the most important components of ecosvstem. which plavs an important role in protecting soil and preventing its rise. So, monitoring and evaluating its changes in nlanning to control the dust storm is effective. The purpose of this studv was to investigate the changes of vegetation in the internal and external dust storm sources of Kermanshah province and dust extension areas. For this purpose, horizontal visibility data were obtained from the meteorological administration of Kermanshah in the statistical period 2005 to 2015. In 2008 and 2009. as well as in the months of Mav. June and Julv. due to the most freauent occurrence of days with dust were the basis of study. In the next step, the most important events of dust in the vears and months were selected based on two criteria of minimum horizontal visibilitv and maximum duration of continuity and the MODIS satellite image (MOD02) was obtained for them. The BTD method was used to detect dust storm. Then vegetation changes were evaluated using MOD09 Q1 MODIS and NDVI index in three vear intervals and in good. moderate and poor vegetation categories. The results of this study showed that in 2006 the area of good vegetation class in the external and internal dust storm sources was $1.8 \%$ and $28.25 \%$. respectivelv. but in 2015 the area of this class decreased in both external $(0.87 \%)$ and internal $(18.62 \%)$. In general, considering the important role of vegetation in soil conservation, it can be taken with the conservation. regeneration and extension of the vegetation. especiallv in areas where the results of this research have been reduced, have taken an effective step in controlling the dust storm.
\end{abstract}

Keywords: Vegetation, MOD09 Q1, NDVI, Dust storm sources, Brightness temperature difference 